\title{
Fatigue Studies on Composite Eliptic Spring Elements for Automotive Front Suspension
}

\author{
* Subramanian $\mathrm{M}^{1}, \mathrm{~J}_{\text {NNiresh }}^{2}$ \\ \{sriisubbumani@gmail.com, nireshcbe@gmai.com\} \\ Associate Professor, Department of Automobile Engineering PSG College of Technology, \\ Coimbatore, Tamil Nadu, India, ${ }^{1 *}$, Assistant Professor , Department of Automobile Engineering PSG \\ College of Technology, Coimbatore, Tamil Nadu, India ${ }^{2}$
}

\begin{abstract}
Composite Elliptic spring elements were subjected to fatigue studies. A common trend of mild stiffness reduction during the initial stages of fatigue tests followed by a higher stiffness reduction rate during the intermediate stage (103-105 cycles) was observed. Above 105 cycles the stiffness reduction was found to be less severe. The fatigue performance of the spring elements having UDR configuration was always found to be poor compared to the ones having UDR/WRM laminated type-1 configurations. The spring elements having CTBN modified matrices were found to give superior fatigue performance than the unmodified matrix spring elements. The fatigue studies reveal that the upper limit of the deflection should be only $25 \mathrm{~mm}$. At $30 \mathrm{~mm}$ deflection levels the stiffness reduction of both the spring elements were observed to be very severe. The spring element having $15 \%$ CTBN modified matrix and laminate type- 1 configuration gave the best results with respect to the fatigue characteristics. The stiffness reduction at the end of 1 million cycles was found to be $22 \%$..
\end{abstract}

Keywords: UDR/WRM, CTBN.

\section{Introduction}

One of the most important requirements of any automotive suspension system is good fatigue performance. It is well known that the optimally tailored polymer matrix composites can very well match or can give better fatigue performance than their isotropic counterparts. Generally, polymer matrix composites have multiple modes of fatigue failure. Major types of composite fatigue failure mechanisms can be listed as follows.
i. Fibre/matrix debonding
ii. Matrix cracking
iii. Fibre breaking.
iv. Delamination among the plies.

In addition to the overall magnitude of stress, these fatigue failure mechanisms are also dependent upon the laminate stress intensity factor. Though, these failures occur at different conditions, the combined effect of all these factors would lead to the reduction in stiffness and degradation in the structural integrity of the composite. However, a proper study on the fatigue damage and damage mechanisms could offer a wider scope for the enhancement of fatigue performance significantly. Normally the fatigue performance of the composites can be improved in the following ways.

i. By using a matrix of higher toughness. 
ii. By using a suitable reinforcement fibres.

iii. By improving the adhesion between fibre and matrix.

iv. By optimizing the laminate configuration.

Reliable evaluation and analysis of fatigue performance of any composite requires exact assessment of the following.

i. Overall fatigue damage caused.

ii. Type of fatigue failure and rate of fatigue damage progression throughout its fatigue life.

The overall fatigue damage to a structure is generally expressed in many ways such as by S-N plots, modulus degradation plots and by using suitable mathematical models. The latter (type and rate of damage progression) can be

\section{Basic Approach}

Different types of composite elliptic spring elements (variables: matrix composition and laminate type) were subjected to displacement-controlled fatigue studies. These studies were carried out in a specially designed fatigue testing machine. In the present study, the fatigue damage to the spring element is expressed in terms of percentage stiffness reduction, which is calculated as follows.

$$
\begin{aligned}
& \text { Stiffness reduction }(\%)=1-\frac{E_{n}}{E_{0}} \times 100 \\
& \text { where } \\
& \quad E_{n}-\text { - Stiffness after 'n' number of cycles } \\
& E_{0} \text {-- Original or initial stiffness }
\end{aligned}
$$

The stiffness reduction was measured at regular intervals. From these observations, the influence of different variables on the fatigue performance of the spring element has been investigated.

\section{Experimental Procedure}

This section describes the details of the experiments carried out and the experimental procedure followed.

Fabrication of the composite Spring Element

To perform the fatigue sufficient number of different types (matrix composition and laminate type) of spring elements were fabricated by filament winding method. The fabrication of elliptic spring element requires proper design and fabrication of a suitable elliptic mandrel. static design stiffness of each spring element was $150 \mathrm{~N} / \mathrm{mm}$. This length of the semi-minor axis was fixed as $5.0 \mathrm{~cm}$ based on the actual dimensions of passenger car suspension spring. Based on this the length of the semi-major axis was fixed as $7.5 \mathrm{~cm}$. Using these dimensions, the mandrel for the elliptic spring elements were fabricated.

Before starting the filament winding process, the elliptical surface of the mandrel was wrapped with a fine $(0.3 \mathrm{~mm}$ thick) glass reinforced Teflon fabric in order to facilitate the easy removal of the cured spring element. Unidirectional Roving (UDR) composite elliptic spring elements were fabricated by filament winding process. Continuous UD rovings were wound 
around the mandrel until the desired spring thickness was attained. In the case of springs having unidirectional/woven roving mat (UDR/WRM) type laminate, the WRM was properly cut and wound. A sufficient overlap at the ends was maintained. As far as the fabrication of UDR/WRM type laminate spring is concerned the UDR and WRM laminae were wound as alternate layers owing to the following:

i. The UDR lamina gives higher stiffness and strength to the spring for a given thickness.

ii. The UDR and WRM were filament wound alternatively in order to improve the interlaminar shear strength (ILSS) of laminates.

Then it was removed and kept in the air circulating oven at $120^{\circ} \mathrm{C}$ for 4 hours. In the case of the piperidine cured composite spring elements, the mandrel was allowed to rotate in between two Infra-red (IR) lamps one on the either side of the mandrel until the gelation is completed.

Fatigue Tests

To carry out the fatigue studies, a special fatigue testing set up was designed and fabricated. This fatigue testing set-up consists of a 3-phase AC motor, a reduction gear box and fixtures for fixing the elliptic spring element. The composite spring element was subjected to flexural fatigue type of load. The spring element was loaded along its minor axis. The fatigue load was applied through an eccentric (deflection-controlled fatigue test).

The eccentric is driven by the output shaft of the reduction gear box. The gear reduction is 1:40. The reduction gear box is connected to the electric motor. The fixture for elliptic spring element has been designed and fabricated in such a way that there could be no lateral movement of the spring element at the time of loading. Two vertical guide pins were provided to ensure this. The spring element was suitably clamped to a rigid fixture at the other end (bottom) of the minor axis, along its width of the spring.

The fatigue test parameters are:

Displacement: 30 and $25 \mathrm{~mm}$

Frequency $: 0.6 \mathrm{~Hz}$

Loading: Cyclic fatigue (deflection controlled)

In the case of automotive suspension system, the typical frequency ranges from 30 to 40 Hz. Though the experimental frequency $(0.6 \mathrm{~Hz})$ used was low, it has specific practical advantages such as:

(i) When the vehicle encounters a bump or pothole, the speed has to be reduced. This will reduce the frequency of the suspension system considerably. So, the amplitude and frequency of the actual vehicle suspension system can become closer to the experimental amplitude and frequency.

(ii) Since the frequency is low strain rate will also be low. Due to this sufficient time will be available for the actuation of toughening mechanisms of Carboxyl terminated butadiene $(\mathrm{CTBN})$ modified matrices. This will help in evaluating the efficiency of the rubber (CTBN) addition, in enhancing the fatigue performance of the spring elements

The fatigue damage progression rate (at the amplitude level used in the present study) will be higher, if the frequency is higher. This disadvantage gets eliminated at the present frequency level. Table 1 gives the details of the fatigue studies carried out on different spring elements. 
Table 1 Details of fatigue tests

\begin{tabular}{||l|l|l|l|l||}
\hline $\begin{array}{l}\text { Type of } \\
\text { spring } \\
\text { (laminate } \\
\text { type })\end{array}$ & $\begin{array}{l}\text { CTBN } \\
(\%)\end{array}$ & $\begin{array}{l}\text { Deflectio } \\
\mathrm{n} \\
(\mathrm{cm})\end{array}$ & $\begin{array}{l}\text { No. of } \\
\text { cycles } \\
\left(\mathrm{x} 10^{3}\right)\end{array}$ & $\begin{array}{l}\text { No. of } \\
\text { samples } \\
\text { used }\end{array}$ \\
\hline UDR & 0 & 3 & 300 & 2 \\
UDR & 15 & 3 & 300 & 2 \\
UDR/WRM & 0 & 3 & 300 & 2 \\
UDR/WRM & 15 & 3 & 300 & 2 \\
\hline UDR/WRM & 0 & 2.5 & 600 & 3 \\
UDR/WRM & 15 & 2.5 & 600 & 3 \\
\hline
\end{tabular}

The fatigue studies were carried out in two phases. During the first phase $30 \mathrm{~mm}$ displacement was used. After analysing the results of the first phase, the second phase experiments were carried out with $25 \mathrm{~mm}$ displacement. Each experiment was repeated with either 2 or 3 -samples.

\section{Results And Discussion}

In this section, the results of the fatigue studies on different type of composite elliptic springs elements are presented. Initially first phase of the experiments was carried out and their results have been discussed. The first phase of experiments was carried out, mainly to determine the following.

i. Whether the stiffness reduction is within the allowable limits or not.

ii. The type of the laminate configuration to be used for further fatigue testing.

The observed variation in spring stiffness with fatigue cycles for spring elements having configurations of UDR laminate and UDR/WRM laminate type-1 (the configuration of the laminate-1 is given in chapter-2) is illustrated in Fig. 1 Both the spring elements were made using the unmodified matrix. 


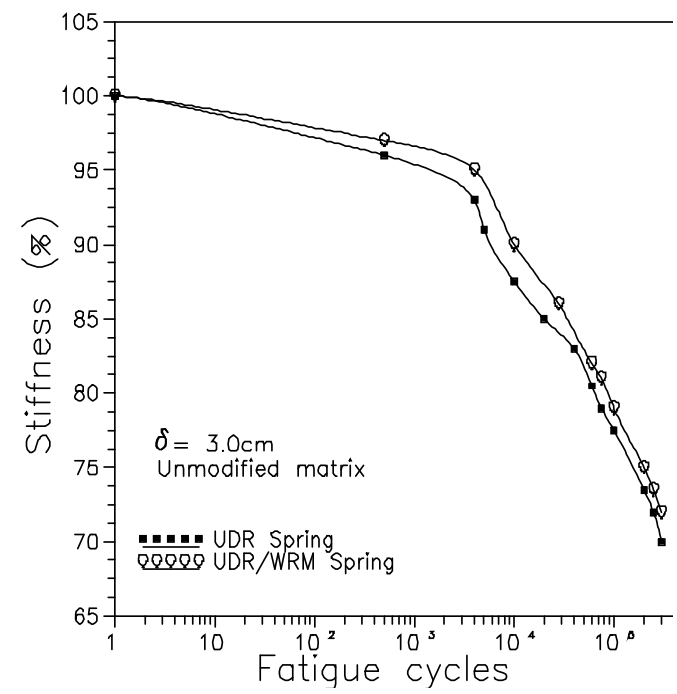

Fig. 1 Fatigue characteristics of different spring elements made using unmodified matrix.

The Fig. 2 gives the same results of Fig. 1 in a linear $\mathrm{x}$-axis. The Fig 2 has been provided in order to present a clear picture of the fatigue characteristics during the later or closing stages of the fatigue tests. The log scale (x-axis) graphs present the initial stages (i.e up to 10,000 cycles) of fatigue behaviour of spring element quite clearly. So, both linear and log axis graphs have been given in order to have a better understanding of the fatigue performance of the spring elements.

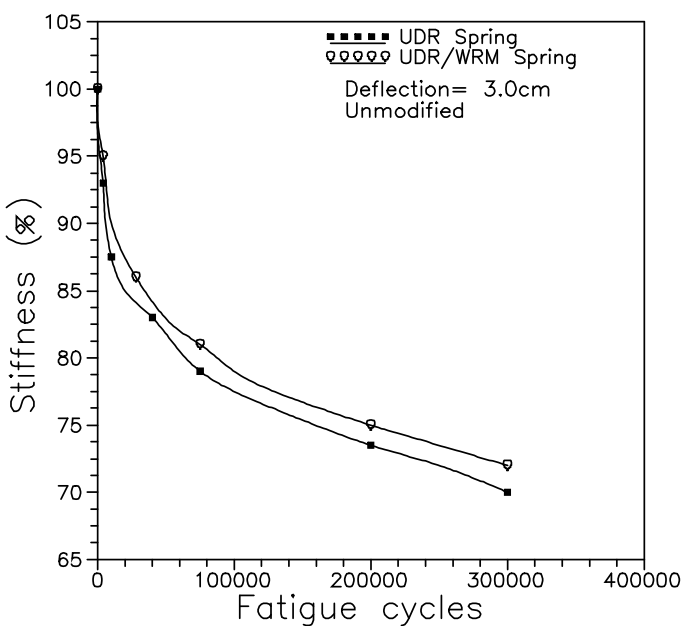

Fig. 2 Fatigue characteristics of differentspring elements with unmodified matrix presented in linear $\mathrm{x}$-axis

The stiffness reduction is minimal up to 1000 cycles for both type of spring elements. The composite laminate may contain many weak spots due to the fabrication defects. These weak spots could act as focal spots for crack initiation from the very beginning of the fatigue life itself. 
With each cycle of loading, the fatigue damage increases. In addition to the failure at the weak spots, the debonding at the fibre/matrix interface can also lead to stiffness reduction. Debonding can be regarded as equivalent to the crack initiation. The fibre/matrix debonding could be followed by or associated with the matrix cracking. The debonding reduces the load carrying capacity of the reinforcement at particular region. As a result of this, the other regions of the laminate can be subjected to higher stresses which may lead to subsequent fatigue failure.

A gradual decrease in the rate of stiffness reduction during the later stages of fatigue cycling can be noticed from the Fig. 2 . The decrease in the stiffness reduction rate could be attributed to the following:

i. Depletion of crack or damage initiation sites.

ii. Crack arresting and crack deflection by the reinforcement material.

UDR and laminate- 1 type spring elements retain $70 \%$ and $72 \%$ of their original stiffness respectively, at the end of $3.0 \times 10^{5}$ cycles. The better performance of UDR/WRM laminate type-1 spring elements can be attributed to its greater ability to confine or arrest the propagating cracks.

The stiffness reduction with fatigue cycles for spring elements having $15 \%$ CTBN modified matrices is presented in the 3 At the end of $2.5 \times 10^{5}$ cycles, the stiffness of the modified UDR laminate and UDR/WRM laminate type-1 spring elements are found to be $73 \%$ and $75 \%$ respectively. Comparing the laminate type- 1 spring elements having unmodified and modified matrices, the later retains $75 \%$ of its original and the former retains $73.5 \%$ of its original stiffness at the end of $2.5 \times 10^{5}$ cycles.

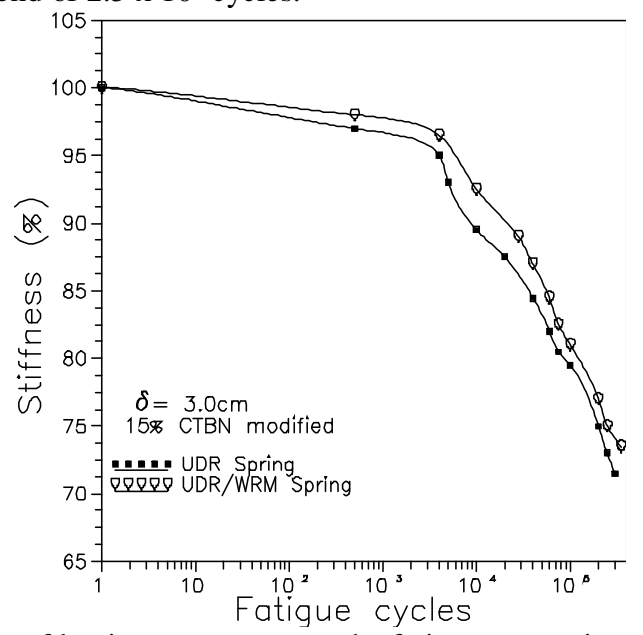

Fig. 3 Influence of laminate structure on the fatigue properties of spring elements

The influence of CTBN addition on the fatigue properties of UDR/WRM laminate type-1 spring elements is shown in the Fig. 4. It can be observed that throughout the fatigue test range, the spring element with modified matrix exhibits better fatigue properties than the unmodified one. However, this improvement is only marginal and can be attributed to the presence of tougher matrix. 


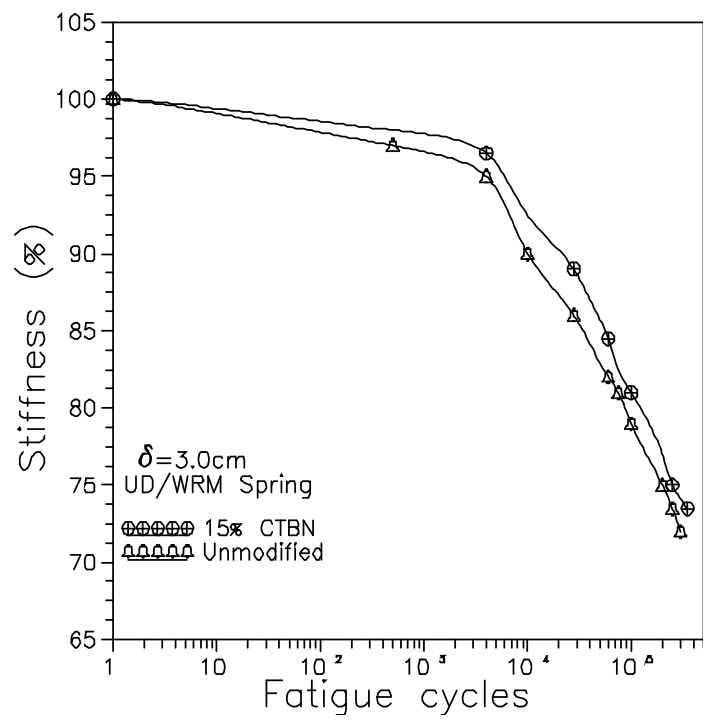

Fig. 4 Influence of CTBN toughening onthe fatigue properties on spring elements

The results of the fatigue studies with $30 \mathrm{~mm}$ deflection show that the best performance was obtained for the 15\% CTBN modified UDR/WRM laminate type-1 spring element. However, the magnitude of stiffness reduction was found to be quite high. In order to reduce the magnitude of fatigue damage the displacement was limited to $25 \mathrm{~mm}$. Further fatigue studies were conducted keeping the deflection as $25 \mathrm{~mm}$. This constitutes the second phase of fatigue studies.

\section{Results of Second Phase Fatigue Studies}

The fatigue characteristics of UDR and UDR/WRM laminate type-1 spring elements have been presented in the Fig. 5 and 6 Similar to first phase experiments, here also laminate type-1 spring elements give superior fatigue performance. In the case of laminate type-1 spring elements, the initiated crack can be arrested or branched off when they meet obstacles such as glass fibres. Further, branching or deflection of the crack can lead to diffusion of the crack-tip stress and consequently to crack arresting. Because of this, the cracks do not propagate to long distances and could be confined to a ply in which they were initiated. Due to this the UDR/WRM laminate can retain a good strength even after the formation of many cracks.

In UDR laminate spring, continuous fatigue cycling could increase the crack density, crack size and consequent laminate degradation. Also, in UDR laminate configuration, it is possible that the crack formed can grow and propagate to other plies causing a severe deterioration in the stiffness. As a result of all these factors the UDR spring element undergoes a comparatively severe reduction in stiffness. 


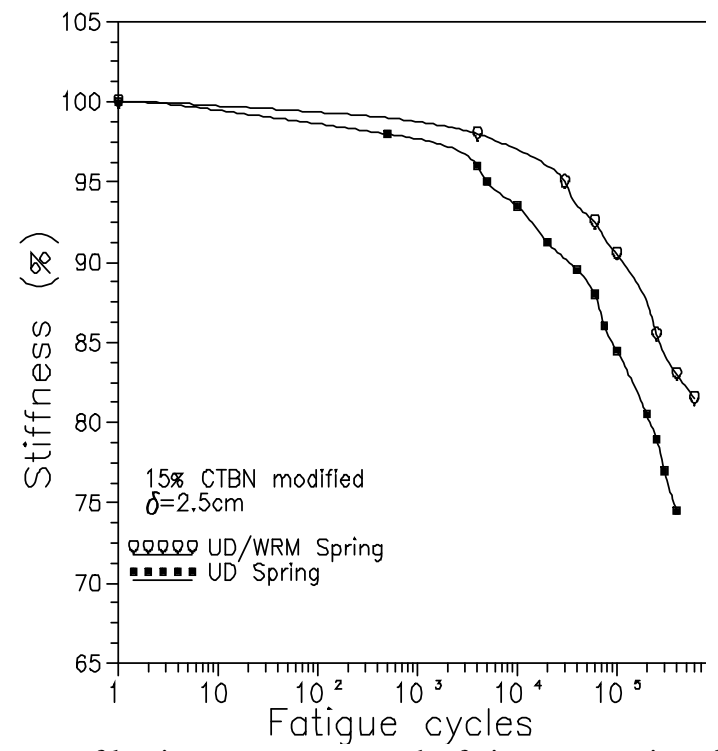

Fig. 5 Influence of laminate structures on the fatigue properties ofspring elements.

At the end of $4.0 \times 10^{5}$ cycles, the UDR laminate spring element has lost $25 \%$ of original stiffness whereas UDR/WRM laminate type-1 spring has lost $17.0 \%$ of original stiffness. The Fig. 6 illustrates the fatigue behavior of different spring elements during the initial stages of fatigue tests (upto $1.0 \times 10^{5}$ ) cycles in linear $\mathrm{x}$-axis.

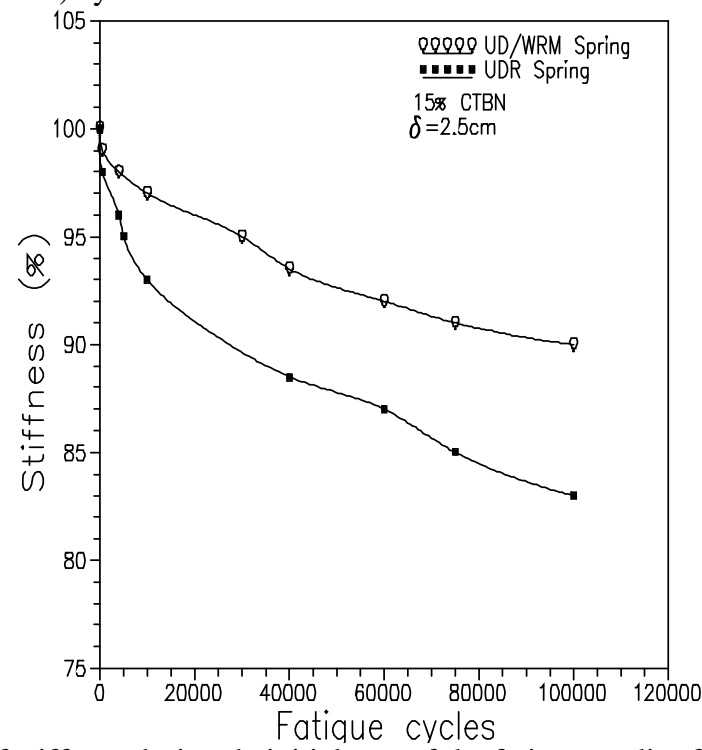

Fig. 6Variation of stiffness during theinitial part of the fatigue studies for spring elements with different laminate structure 
The effect of CTBN addition on the fatigue characteristics of the composite elliptic spring elements is illustrated in the Fig. 7. The fatigue properties of unmodified and $15 \% \mathrm{CTBN}$ modified UDR/WRM laminate type-1 spring elements have been compared. At the end of 6.0 x $10^{5}$ cycles, the CTBN modified spring element results in $18.5 \%$ stiffness reduction. This is the best possible performance obtained among all the studies conducted.

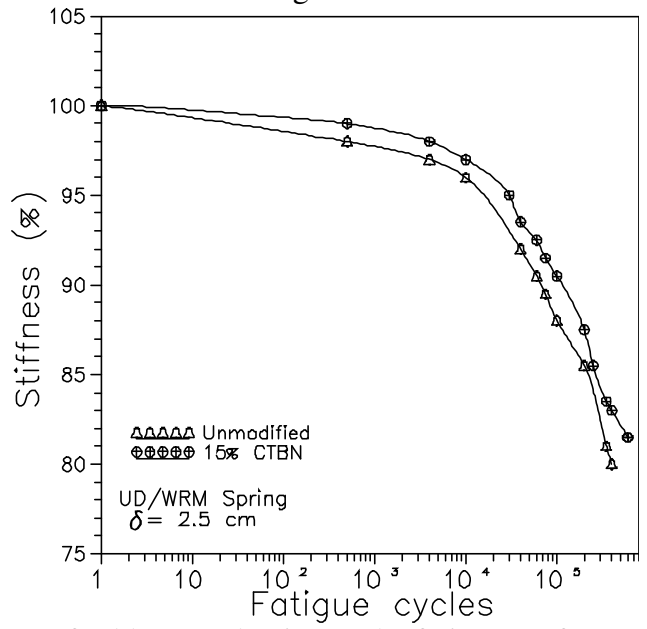

Fig. 7 The influence of rubber tougheningon the fatigue performance of spring elements at a deflection of $25 \mathrm{~mm}$.

In order give a clear picture of fatigue performance of spring elements, the stiffness reduction with fatigue cycles has been presented in table 2 .

Table 2. The stiffness reduction of UDR/WRM laminate type-1 spring element having $15 \%$ CTBN modified matrix during fatigue cycling.

The superior fatigue performance of CTBN modified composite spring element can be explained as follows:

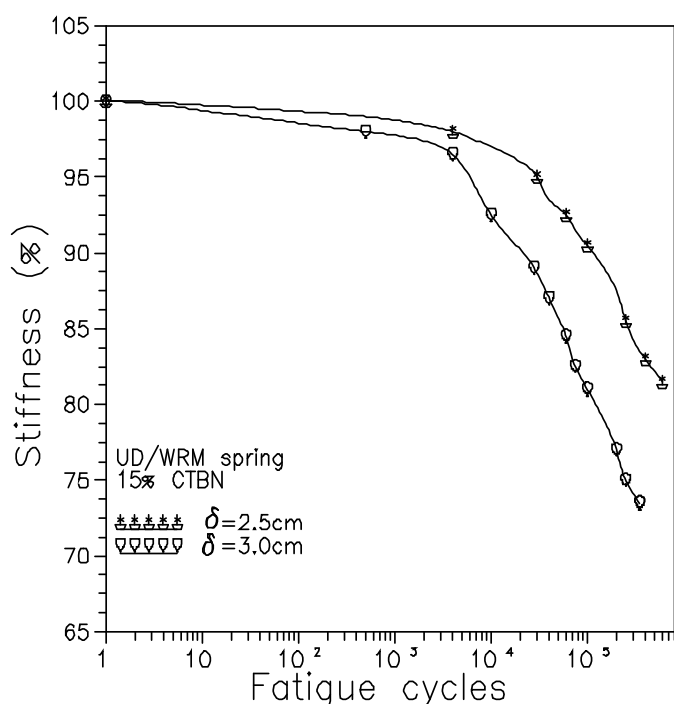


Fig. 8 The influence of fatigue deflectionamplitude on fatigue performanceof the spring elements

\begin{tabular}{||l|l||}
\hline $\begin{array}{l}\text { No. of cycles } \\
\text { Deflection }=2.5 \mathrm{~cm}\end{array}$ & Stiffness Reduction (\%) \\
\hline $10 \times 10^{3}$ & 3.0 \\
$100 \times 10^{3}$ & 9.5 \\
$250 \times 10^{3}$ & 14.5 \\
$400 \times 10^{3}$ & 17.0 \\
$600 \times 10^{3}$ & 18.5 \\
\hline
\end{tabular}

The influence of the magnitude of deflection (amplitude) on the fatigue properties, has been presented in Fig. 8. Higher stiffness reduction was observed in the case of the spring element subjected to $3.0 \mathrm{~cm}$ deflection, compared to the $2.5 \mathrm{~cm}$ deflection.

The spring has been designed for a spring stiffness of $100 \mathrm{~N} / \mathrm{mm}$. The projected or extrapolated stiffness reduction was $22 \%$ after $10^{6}$ cycles. This reduction is quite high as far as the functional requirement of the spring element is concerned. This reduction can be compensated by suitable design considerations or modifications. The spring element can be designed for an initial stiffness of $150 \mathrm{~N} / \mathrm{mm}$. So, the reduction of $22 \%$ spring stiffness will result in $33 \mathrm{~N} / \mathrm{mm}$ stiffness reduction after $10^{6}$ cycles. However, this prediction is based on the assumption that the spring element will behave alike for both 100 and $150 \mathrm{~N} / \mathrm{mm}$ stiffness. The final stiffness after $10^{6}$ cycles will be about $117 \mathrm{~N} / \mathrm{mm}$.

Ways of increasing the stiffness of composite spring elements are:

(i) Increasing the thickness of the spring.

(ii) Increasing the width of the spring.

\section{Summary Of The Results}

This section gives the summary of the results of the fatigue, acoustic emission and free vibration studies conducted. A common trend of mild stiffness reduction during the initial stages of fatigue tests followed by a higher stiffness reduction rate during the intermediate stage $\left(10^{3}-10^{5}\right.$ cycles) was observed. Above $10^{5}$ cycles the stiffness reduction was found to be less severe. The fatigue performance of the spring elements having UDR configuration was always found to be poor compared to the ones having UDR/WRM laminated type-1 configurations.

The spring elements having CTBN modified matrices were found to give superior fatigue performance than the unmodified matrix spring elements. The fatigue studies reveal that the upper limit of the deflection should be only $25 \mathrm{~mm}$. At $30 \mathrm{~mm}$ deflection levels the stiffness reduction of both the spring elements were observed to be very severe. The spring element having $15 \%$ CTBN modified matrix and laminate type-1 configuration gave the best results with respect to the fatigue characteristics. The stiffness reduction at the end of 1 million cycles was found to be $22 \%$. 


\section{References}

[1] Gill, P.S. and J.N.Leckenby (2014) Dynamic mechanical analysis of advanced composites. Composite Structures, 2, 235-243.

[2] Gledhill, R.A., A.J.Kinloch, A.Yamini and R.J.Young (2011) Relationship between mechanical properties of and crack propagation in epoxy resin adhesives. Polymer 19, 574-582.

[3] Guild, F.J., D.Walton, R.D.Adams and D.Short (2015) An application of acoustic emission to fibre-reinforced composite materials. Composites, 173-179.

[4] Hahn, H.T. and R.Y.Kim (1998) Fatigue behaviour of composite laminates, J. Composite Materials, 10, 156-180.

[5] Hajela, P. and C.J.Shih (2018) Optimum synthesis of polymer matrix composites for improved internal material damping characteristics. 26, AIAA Journal, 504-506.

[6] Hamstad, H. (2012) Acceptance testing of graphite epoxy composite parts using an acoustic emission monitoring technique. NDT International, 307-314.

[7] Hamstad, M. A and T.T.Chiao (2002) Acoustic emission produced during burst tests of filament wound bottles. J.Composite Materials, 7 320-332.

[8] D. S. Vijayan and J. J. Daniel, "An investigation on the torsional effect of symmetric moment resisting frame system subjected to eccentric reinforced concrete lift wall - A finite element approach," Int. J. Eng. Trends Technol., vol. 69, no. 8, pp. 179-184, 2021, doi: 10.14445/22315381/IJETT-V69I8P222 